\title{
PROVIDING A TECHNICAL SOLUTION TO IMPROVE NURSES' PERFORMANCE IN DRUG DELIVERY: A QUALITATIVE STUDY
}

\author{
Alireza Shahsavani 1 , Fateme Kian ${ }^{*}$ Anahita Saraband ${ }^{2}$ \\ 1. Community Nursing Research Centre, Zahedan University of Medical Sciences, Zahedan, Iran \\ 2. Dental Student, Zahedan Univ ersity of Medical Sciences, Zahedan, Iran
}

Correspondence:Fatemeh Kiani, fkiani2011@yahoo.com

\begin{abstract}
BACKGROUND AND PURPOS:

Technical measures were taken to improve the performance of nurses in the drug delivery process in hospitals affiliated to Zahedan University of Medical Sciences in Iran

\section{MATERIALS AND METHODS:}

This study was conducted with a qualitative approach and contractual content analysis method in 2017-18. Participants were 16 nurses and a physician working in different wards of the hospital who were included in the study by purposive sampling. The method of data collection was semi-structured interviews. All interviews were conducted and reviewed and analyzed by contractual content analysis.
\end{abstract}

\section{RESULTS:}

In the present study, 'steps to prevent drug error' as the main category and 'acting professionally' and "providing technical solutions' as two subcategories were extracted from the data.

\section{CONCLUSION:}

The results showed that the use of nurses 'experience and knowledge as technical solutions to prevent drug errors can play an important role in improving nurses' performance and increasing patient safetykeywords

\section{KEYWORDS:}

Performance Improvement, Drug, Nurse, Qualitative Study

\section{INTRODUCTION}

Maintaining the standards and performance of nursing services in quality and promoting patient health and safety is undeniable [1] Every year, medication errors can pose a serious risk to patients' health and pose a threat to their

safety; According to the findings of studies, tens of thousands of patients die annually as a result of accidents and errors of treatment staff in various treatment situations. [2] The American Nurses Association recognizes the roles of nurses in maintaining the health of various body systems, cooperating and coordinating with other treatment 
systems, and performing activities based on law and regulations, taking into account the health of the community [3] Nurses, as a manager, have a directrole in ensuring the safety of patients [4] because about $40 \%$ of the nurse's clinical activities in the hospital are spent on medication. [5] A professional nurse is a committed and responsible person who acquires the necessary professional skills with special training. [6]

Medication is one of the basic measures of nursing and takes up approximately $40 \%$ of the nurse's working time. [7] Studies conducted in Iran on drug error provide different statistics. In their study, Shams et al. stated that nurses' errors were $28.9 \%$. [8] In the study of Mirzaei et al. nurses reported medication error of $79.2 \%$.[9]

Nurses spend most of their time with patients and monitor them throughout the medication process from the time they are admitted to the hospital until they are discharged. [10] Nurses are responsible for the direct care of patients and participate in the process of giving them medication. This can lead to drug errors by them. [11]

Nurses with high moral and professional commitment will strive to achieve professional values, one of the most important examples of which is the reduction of medication errors as one of the most important indicators of patient safety. [12] In the workplace, organizational commitment of employees is one of the important organizational variables that affects their job performance and behaviour. [13] The results of the study by Wolf et al. showed that $20 \%$ of all errors that occur in the medical field, including $39 \%$ related to physicians' errors in prescribing, $38 \%$ related to nurses drug use and and $12 \%$ related to pharmacists $11 \%$ is related to the stage of transferring the doctor's order from the file to the pharmacopoeia. [14] In the study of Mirzaei et al., A review of the literature shows that approximately $65-87 \%$ of medication errors occur in the drug delivery process. Although it seems that medication errors are known, we still see medication errors in various forms in clinical wards. Therefore, the present study was conducted with the aim of providing a technical solution to improve nurses' performance.

\section{MATERIALS AND METHODS}

\section{STUDY DESIGN AND PARTICIPANTS}

This qualitative study was conducted on 17 participants (16 nurses and 1 physician) in an educational hospital of
Zahedan University of Medical Sciences from 10 August 30 , 2019 to 30 March 2020. This hospital employs 52 nurses. Participants were selected using a purposive sampling method. The maximum variation sampling technique was used for selecting the participants with different experience to collect rich data. The inclusion criteria included having a BSC degree and having 2 years' work experience. The COREQ checklistwas used for the study report [7]

\section{DATA COLLECTION}

First, the researcher explained the study's aim for the participants. After getting written consent from the participants, the data were gathered by individual face-toface semi-structure interviews in a comfortable room in a ward at the hospital. For convenience, only the researcher and the participant were in the room. Some of the interview questions were:

1. Describe how medication is prescribed during a shift.

2. What do you do when there is a medication error?

3. What are the factors causing medication errors?

4. How can medication errors be prevented?

Participants were interviewed one at a time and four participants had a second interview to complete the first one. The duration of the interview was 30-60 min. All interviews were tape recorded using a digital voice recorder. Also, data was collected using field notes.

\section{DATA ANALYSIS:}

The researchers typed all the recorded interviews for subsequent analysis. Data analysis was conducted using Braun and Clarke's [15] six-stage thematic analysis method. The six stages included: 1. Familiarization with the data, 2. making preliminary codes using systematic analysis with codes having the same meaning placed alongside each other, then collating codes across transcripts, 3. searching for themes with codes having the same meaning categorized under the same theme, then assigning a label, (4) reviewing themes, checking extracted codes and text, considering the need for needed themes, and developing a thematic map of analysis, (5) defining and naming themes with refinement and definition to accurately tell the story, and (6) producing the final report with the clear explication of linked themes. [15] Software MAXQDA V12 was used to manage and code the data.

Transcripts of the interviews were returned to the participants for their feedback. Lincoln and Guba's 
trustworthiness criteria were used to ensure the accuracy of findings and include credibility, transferability, dependability, and conformability. To check the credibility the researchers were dealing with the data continuously for about 2 years; also, credibility was checked through member check, long-term interview, immersion into interview data, and consensus of codes between researchers.

To check the data trustworthiness, the data were given to a small group of nurses who were familiar with clinical environments but who did not participate in the present study. So results were shared with them and their feedback were collected. To ensure the dependability of the study, a step-by-step repetition and precise inspection which included accurate investigation of relevant data and documents was done by an external reviewer. Finally, conformability (objectivity) was verified with the use of an external reviewer who followed the audit trail created during the study. [16]

Ethical consideration: This study was approved by the Ethics Committee of Zahedan

University of Medical Sciences in Iran (ethic code: IR.ZAUMS.REC.1395.165). Before data collection, the researcher explained the full details about the study. If individuals were interested in participation, written informed consent was obtained. Participants were also assured that their information will be kept confidential. Audio files were deleted to protect patients' information after taking notes and analyzing them.

\section{RESULTS}

Sixteen nurses and one physician participated in this study. Their minimum age was 25 and maximum was 50 years. Their work experience was 3-25 years. 10 were married and six were single.

From the data analysis, two main categories were extracted: a category of 'error prevention steps' with the subcategories of 'acting professionally' and 'providing technical solutions'.

\section{PROVIDE TECHNICAL SOLUTIONS:}

"Practicing professionalism" and "providing technical solutions" were identified.
Some nurses believed that medication should be given by an experienced nurse, in which case medication error would be reduced. A nurse who worked in the pediatric ward said:

'In the pediatric ward, one should be very careful when giving medicine, for example, the dose of the medicine should not be mistaken. Therefore, the nurse in charge of medicine is an experienced and skilled nurse and inexperienced nurses work with him. For this reason, no error has occurred so far, or before the error occurred, especially in the dosage of the drug, the drug error was prevented.' '... In the CCU, all drugs are injected intravenously. We have no intramuscular drug at all. 'According to the conditions of the patients in this ward, the nurse who is supposed to work in this ward must be instructed in prescribing the medications to prevent the mistake of prescribing the medication' (Nurse No. 10)

In expressing their experiences in the field of drug error prevention, nurses referred to cases that were related to technical solutions and were factors in preventing drug errors. Items such as identifying high-risk drugs, storing some drugs separately, knowing how to prescribe the drug, using a skilled nurse to administer the drug, and paying attention to accreditation and finding solutions to the error were technical error prevention strategies.

'According to the list of the Ministry of Health, 20 high-risk drugs have been identified in hospitals and labeled red, such as potassium chloride, magnesium sulfate, sodium bicarbonate, lidocaine' said the experienced nurse in reducing medication errors, especially for dangerous drugs. 'Atropine, epinephrine, sodium heparin, haloperidol, propranolol, etc. These drugs are checked by two nurses. This solution reduces the possibility of error in high-risk drugs' (Nurse No. 3)

'Once I took oxytocin instead of neostagmine, but I realized in time. One of the reasons I was wrong was their similarity, but now, to reduce such errors, they have ordered that these drugs be stored separately and possibly, 'said another nurse. 'The error has been reduced to zero.' (Nurse No. 1)

One of the experienced nurses who was in charge of the ward suggested teaching the way to prescribe specialized drugs in the ward as a way to reduce medication errors: 
'... In the CCU ward, all drugs are injected intravenously. The ward to the nurse who is supposed to work in this ward must be instructed to prescribe the drugs to be used to prevent the mistake of prescribing the medicine' (Nurse No. 10)

Some nurses believed that accreditation helped prevent many mistakes. One of the nurses said: 'Currently, there is a report of drug error in the accreditation of medical centers in the measurements provided by the Ministry of Health. This means that we comply with the same 8 international drug laws.' 'Assessment has reduced the percentage of medication errors. '

Nurses also suggested error reporting as one of the ways to prevent errors. The nurse said:

'If there is a medication error the nurse should report the error. In fact, when you report the error through the boxes that are installed in each ward of the hospital. The error is detected and the authorities can look for a solution to this problem and offer a solution to prevent that error. For example, one of the nurses with a long history of work said that one of the problems that always leads to mistakes in giving medicine is that with a few medicines that we have drawn and prepared in the room with a syringe, we go over the patient's head and give medicine. Sometimes it is possible that the drug to be injected in the form of an infusion is given to the patient in the form of a bolus and injected directly, which led to complications which was promptly intervened because the patient was in the intensive care unit, was immediately observed complications that had unfortunate consequences.

Next, the nurse suggested that the medicine be prepared on the patient's bedside as much as possible, and when injecting the medicine, it is better to mention the name of the medicine to the patient, as a result of which, firstly, the nurse pays attention and secondly, they are sensitive that it is better for the patient to be informed about the medication he is receiving In this way, drug errors are prevented.' (Nurse No. 16)

\section{DISCUSSION}

In this study, the process of nurses' exposure to medication instructions and ways to prevent medication error by nurses were explained. From the data analysis, two main categories were extracted: a category of "error prevention steps" with the subcategories of "acting professionally" and "providing technical solutions".

The findings of this study showed what are the technical strategies to improve the performance of nurses and prevent drug errors, which are discussed in each of the following dimensions.

\section{PAY ATENTION TO VALIDATION AND FIND SOLUTIONS TO ERRORS}

Considering that recently in the monitoring systems, the accreditation performance in hospitals has created a change in the drug process, the nurses' opinions in this regard are examined and analyzed.

Some nurses believed that accreditation helped prevent many mistakes. One of the nurses said: 'Currently, in the accreditation of medical centres, drug errors are reported in the measurements provided by the Ministry of Health. This means that we comply with the same 8 international drug laws. Paying attention to this law in accreditation has reduced the percentage of medication errors.' (Nurse No. 10)

Consistent with this finding, Yousefnejad et al[17] in a descriptive-analytical study on the effects of accreditation in hospitals affiliated to Tehran University of Medical Sciences found that the implementation of accreditation program improves safety culture and reduces medication errors and develops staff capabilities. [18]

The expansion of accreditation programs increases public awareness of medical errors and bridges the gap in the development of patient safety in the health care system. [19] According to studies, accreditation programs improve the quality and performance of organizations. [20] Findings of the study by Dekaran and O'Farrell [19] showed that after the implementation of accreditation programs, there was a significant reduction in hospital complaints about drug accidents. [19] Findings from several studies examining the effects of accreditation programs in hospitals in South Africa, Zimbabwe, Australia, Egypt, and the Philippines indicate the positive effects of accreditation programs on reducing patient complaints from health care providers about drug accidents. [21] 
Findings of Mekory et al.'s [22] study showed that the implementation of the accreditation program has significantly reduced medication errors. [22]

\section{READ THE DRUG LABEL:}

'Reading drug labels carefully is a way to reduce errors', said an experienced nurse.

'Reading drug labels reduces the risk of error, but sometimes it becomes careless. For example, years ago I worked in the cardiology department. Warfarin was placed in the place of TNG medicine because both of them are pink. I only paid attention to the color when using it and I made a mistake. The only way to reduce such errors is the nurse's accuracy' (Nurse No. 8)

These types of errors are also called action-based (or slipbased) errors. For example, removing a box of diazepam tablets from a medicine box instead of diltiazem tablets. Findings of one study showed that most medication errors occur due to carelessness and slipping of the nurse during the administration of routine medications. This error can be reduced by carefully reading the drug label, using identifiers such as barcodes, and writing the drug name on the label with a combination of uppercase and lowercase letters. [23]

Findings from the Berman study showed that over the past year, $33 \%$ of drug error reports were related to drug labeling or packaging. [24]

Anderson also found that the use of warning drug labels, with high resolution and quality and with a special colour, significantly reduces the incidence of accidents associated with drug errors. [25]

\section{THE NURSE IS AWARE OF THE LEGAL ISSUES OF MEDICATION ERROR}

Awareness of the nurse about the legal issues of error is one of the characteristics of a nurse acting in a professional manner.

Another experienced nurse mentioned that the nurse's knowledge of legal issues was one of the factors that prevented the error:

'I have had several medication errors. Once I injected streptomycin intravenously instead of intramuscularly. If I told them what they were doing to me, then I did not tell the head nurse, I just checked the patient regularly, and it was difficult, but if I was familiar with the legal issues of error, I would not have suffered so much. I am now informing the ward nurses about medication errors by holding a class' (Nurse No. 8)

In this regard, the findings of a study showed that one of the ways to prevent medication errors is to encourage employees to report errors; In this way, one can think of a way to reduce the error. [26, 27] Therefore, a professional nurse has no hesitation in detecting the error to protect himself from punishment for medication error. While educating nurses, officials can provide a safe environment for nurses to report as much as possible and reduce medication errors. [28, 29]

Based on the findings of their study, Merlin Jolie suggests that continuing education classes on legal issues of nursing errors and keeping pharmacological information up-todate are one of the most important necessities for avoiding medication errors. [30]

\section{FREQUENT TRAINING OF PERSONNEL ON THE IMPLEMENTATION OF THE DRUG PROTOCOL}

Another nurse, who was the head nurse, made it a priority to reduce medication errors. 'We regularly explain to nurses that they should read the medicine label first and prepare the medicine on the patient's bedside and check it again. We have the rule that the medicine label should be checked 2 to 3 times so that it can be checked' he said. 'We do not have anything to report to the supervisor now' (Nurse 7)

The World Health Organization (WHO) states that one of the effective factors in the incidence of nursing errors in the patient's bed is the lack of knowledge and experience of nurses and lack of participation in in-service training courses. [31] Nobahar in a qualitative study found that having knowledge and skills was one of the factors that enable nurses to prevent medication errors. [32]

Consistent with this finding, Taheri et al. in a qualitative study found that one of the most important strategies for preventing drug errors in their view is educating nurses about common drugs in the ward and drug side effects. The existence of authoritative pharmacology books, access to the Internet to medication information, informing nurses about common errors in the hospital, and increasing awareness of prescription and consonant medications were emphasized. [27] 
Many nursing researchers have cited increasing nurses 'pharmacological information as an important strategy to reduce medication errors, and have stated that updating nurses' information about medications, especially new drugs, can be an important factor in reducing medication errors'. [33]

In Iran, the lack of knowledge and awareness of how to implement drug protocols was identified as the most important factor in causing drug errors. [34] To reduce this factor in creating errors, medication information retraining classes can be used for these nurses.

Brady et al. also suggest that in order to calculate the correct amount of medication, teaching mathematics to nursing staff should be considered a necessity. [35]

It seems that by strengthening the curriculum of pharmacology in the undergraduate course, holding continuous training classes and weekly drug conferences within each department according to the specific drugs that are used in each department can increase nurses' awareness of pharmaceutical topics and related equipment.

\section{HAVE ACCURACY}

Another nurse who worked in various departments said about reducing errors:

'There is always an error related to the similarity of the form of the drugs and the names of the drugs and the dosage of the drugs, and it can only be controlled carefully by the nurse. I have experienced this many times when if the accuracy of the ward nurse decreases, an error occurs and this can only be controlled byincreasing the nurse's accuracy' (nurse No. 22)

In confirmation of this finding, Arnson showed that lack of concentration and carelessness in nurses is one of the effective factors in the occurrence of nursing errors. [36] Hashemi et al. 'found that one of the factors associated with the occurrence of nursing errors is individual factors and include unsafe actions arising from Some mental processes of the individual such as forgetfulness, inattention, carelessness and negligence'. [37]

The findings of a study in Australia showed that communication problems with experienced and senior nurses and difficulties in accessing information related to the correct dose of medicationlead to knowledge-based errors and errors due to inaccuracy of the nurse. Therefore, the use of a computer system for prescribing drugs, a barcode-based drug system and re-control by other colleagues can reduce the incidence of errors due to inaccuracy. [37]

\section{IDENTIFY HIGH-RISK DRUGS}

'According to the list of the Ministry of Health, 20 high-risk drugs have been identified in hospitals and labeled with red, such as potassium chloride, magnesium sulfate, sodium bicarbonate, lidocaine, Atropine, epinephrine, sodium heparin, haloperidol, propranolol,' said the experienced nurse in reducing medication errors, especially for dangerous drugs. 'These drugs are checked by two nurses. This solution reduces the possibility of error in high-risk drugs' (Nurse No. 3)

Drugs that potentially harm the patient are called highalert medicine (HAMs) or high-risk medicine. According to The Joint Commission on Accreditation of Healthcare Organizations, or JCAHO, HAM is frequently associated with serious injury to patients or even death. These drugs are dangerous for the patient even when used correctly due to the low therapeutic index. Therefore, it is better to keep them in a separate place with a label identifying high-risk drugs. [25, 38]

\section{USE A SKILLED NURSE IN MEDICATION}

Some nurses believed that medication should be given by an experienced nurse, in which case medication error would be reduced. A nurse who worked in the pediatric ward said:

'In the pediatric ward, one should be very careful when giving medicine, for example, the dose of the medicine should not be mistaken for this reason, the nurse in charge of medicine is one of the experienced and skilled nurses, and inexperienced nurses work with him. For this reason, no error has occurred so far, or the drug error has been prevented before the error occurs, especially in the dose of the drug.' (Nurse No. 4)

According to the Institute for Safe Medication Practices (ISMP), monitoring by an experienced person when prescribing a drug reduces the incidence of drug accidents before the drug reaches the patient by $95 \%$. An experienced nurse is responsible for confirming the correct patient, the correct drug, the correct dose and method of prescribing the drug. [25] 
In connection with this subtheme, Berman (2004) states that performing at least two independent controls at the time of drug administration can reduce medication errors. [24] Tang et al. found that $37.5 \%$ of nursing errors occurred due to novice nurses. [7]

Therefore, it is necessary to think of a plan to use experienced nurses and nurses who have committed less medical errors alongside newly graduated nurses. However, in Iran, as soon as a new nurse is hired, an experienced nurse is hired in another place, such as a nurse supervisor in wards.

\section{FIND SOLUTIONS TO THE ERROR}

Another technical solution that the participants emphasized in order to prevent errors was to find a solution to the error. In this regard, the findings of the study showed that it is important to identify the type of medication error and nurses should be encouraged to report the error. [39]

Findings of the study showed that if the causes of error and its identification are discussed with nurses in meetings, it reduces the probability of error and recurrence of medication error in the future. [40]

Another important way to prevent medication errors is the cooperation of treatment team members, especially nurses and physicians. Establishing communication between physicians and nurses and sharing information can help prevent errors. [40] By identifying the error, in addition to preventing its adverse consequences, the way to prevent it can be discovered.

\section{CONCLUSION}

The purpose of this study was to explain the technical ways to improve nurses' performance from the data analysis, two main categories were extracted: a category of 'error prevention steps'with the subcategories of 'acting professionally' and 'providing technical solutions' .

The findings showed that there is a possibility of medication error for nurses, but if nurses act professionally in performing their duties and have a professional view of technical solutions and improve the nursing performance of control systems such as accreditation they can reduce the likelihood of medication errors by providing technical solutions related to the profession.
It is recommended that in order to improve the performance of nurses to the technical solutions of nurses' performance of the nurse, in-service training should be held seriously and continuously.

The results of this study can predisposing for quantitative and qualitative studies in the fields of management, treatment and education. In particular, qualitative studies are used to reveal intangible aspects of safe drug administration that cannot be identified by quantitative criteria.

\section{SUGGESTIONS FOR FUTURE STUDIES}

Improving the process of prescribing drugs safely in patients: a research action study

Investigating the views of nursing managers regarding the methods of safe drug administration by nurses

Investigating the views of patients regarding the methods of safe drug administration by nurses

Investigating the views of physicians regarding the methods of safe drug administration by nurses

\section{References}

1. Baghcheghi, N. And H.R. Kouhestani, Nursing Students' Errors In Preparation And Administration Of Intravenous Drugs. Strides In Development Of Medical Education, 2008.5(1): P.

2. Arakawa, C., Y. Kanoya, and C. Sato, Factors contributing to medical errors and incidents among hospital nurses --nurses' health, quality of life, and workplace predict medical errors and incidents. Ind Health, $2011.49(3)$ : p. 381-8.

3. Allamzadeh, M., A study on the knowledge of employed nurses in psychiatry unit of hospitals affiliated to Tehran University of Medical sciences about their professional roles. 1992, Dissertation) Tehran: Iran university of Medical Sciences.

4. Yousefi, S.M., et al., Evaluation of Medication Errors and its Related Factors in Kashan Shahid Beheshti Hospital in 2017. 2020.

5. Di Muzio, M., et al., Can nurses' shift work jeopardize the patient safety? A systematic review. Eur Rev Med Pharmacol Sci, 2019.23(10): p. 4507-19.

6. Taylor, C., Potter \& Perry's fundamentals of nursing. 2008: Elsevier Australia. 
7. Tang, F.I., et al., Nurses relate the contributing factors involved in medication errors. Journal of clinical nursing, 2007. 16(3): p. 447-457.

8. Shams, S., et al., Frequency of medication errors and its reporting according to self-report by nurses in hospitals of Khoy city. Nursing And Midwifery Journal, 2012. 10(5): p. 0-0.

9. Derikvand, M. and N. Imanifar, The Relationship between Spiritual Intelligence and Patient Advocacy in Educational Hospitals in Khorramabad 2017. Iranian Journal of Medical Ethics and History of Medicine, 2018. $11(1)$ : p. 278-288.

10. Rohde, E. and E. Domm, Nurses' clinical reasoning practices that support safe medication administration: An integrative review of the literature. Journal of clinical nursing, 2018. 27(3-4): p. e402-e411.

11. Alteren, J., et al., Conflicting priorities: observation of medicine administration. Journal of clinical nursing, 2018.27(19-20): p. 3613-3621.

12. Fathi, A., et al., Survey of medication error by nurses self-reportinintensive care unit of Imam Khomeini hospital-Tehran. International Research Journal of Applied Basic Science, 2014.8: p. 1726-32.

13. Bayazidi, S., et al., Medication error reporting rate and its barriers and facilitators among nurses. Journal of caring sciences, 2012. 1 (4): p. 231.

14. Wolf, Z.R., R. Hicks, and J.F. Serembus, Characteristics of medication errors made by students during the administration phase: a descriptive study. Journal of Professional Nursing, 2006.22(1): p. 39-51.

15. Braun, V. and V. Clarke, Using thematic analysis in psychology. Qualitative research in psychology, 2006. 3(2): p. 77-101.

16. Lincoln, Y.S. and E.G. Guba, But is it rigorous? Trustworthiness and authenticity in naturalistic evaluation. New directions for program evaluation, 1986. 1986(30): p. 73-84.

17. Yousefnejad, K., T. Ziaee, and M. Fazli, Assessment the clinical and paraclinical effects of enhanced external counter pulsation therapy in patients with coronary artery disease. International Journal of Medical Investigation, 2015.4(2): p. 245-248.

18. Mosadeghrad, A.M., A. Akbari Sari, and T. Yousefinezhadi, Evaluation of accreditation effects in hospitals. Tehran University Medical Journal TUMS Publications, 2019.76(12): p. 804-812.
19. Devkaran, S. and P.N. O'Farrell, The impact of hospital accreditation on clinical documentation compliance: a life cycle explanation using interrupted time series analysis. BMJ open, 2014.4(8).

20. Lanteigne, G., Case studies on the integration of Accreditation Canada's program in relation to organizational change and learning: the Health Authority of Anguilla and the Ca'Focella Ospetale di Treviso. Montréal: Faculté de médicine, Université de Montréal, 2009.

21. Alkhenizan, A. and C. Shaw, Impact of accreditation on the quality of healthcare services: a systematic review of the literature. Annals of Saudi medicine, 2011.31 (4): p. 407-416.

22. Mekory, T.M., et al., The proportion of errors in medical prescriptions and their executions among hospitalized children before and during accreditation.

International Journal for Quality in Health Care, 2017. 29(3): p. 366-370.

23. Mira, J.J., et al., A systematic review of patient medication error on self-administering medication at home. Expert opinion on drug safety, 2015. 14(6): p. 815-838.

24. Berman, A., Reducing medication errors through naming, labeling, and packaging. Journal of medical systems, 2004.28(1): p. 9-29.

25. Anderson, P. and T. Townsend, Preventing high-alert medication errors in hospital patients. American Nurse Today, 2015. 10(5): p. 18-23.

26. Oladi Ghadikalaee, R., H. Ravaghi, and S. Hesam, Study Of Nurses' Perceptions On Medication Errors In Pediatric Hospitals In Tehran, Iran. Journal of Payavard Salamat, 2015.9(3): p. 315-328.

27. Tehrani, H., Strategies to prevent medication errors by nurses: A qualitative study. Iranian Journal of Medical Ethics and History of Medicine, 2015.8(3): p. 62-76.

28. FARZI, S., et al., NURSES'EXPERIENCES OF MEDICATION ERRORS. 2014.

29. Madadi, Z. and E. Jaafaripooyan, Nursing Medication Errors, Causes and Solutions. J of Hospital, 2015. 3(14): p. 101-9.

30. Jolly Johnson, M.T., Medication errors: knowledge and attitude of nurses in Ajman, UAE. 2013.

31. Organization, W.H., Technical series on safer primary care. 2017: World Health Organization. 
32. Nobahar, M., Professional errors and patient safety in intensive cardiac care unit. Journal of Holistic Nursing and Midwifery, 2015. 25(3): p. 63-73.

33. Alrabadi, N., et al., Medication errors among registered nurses in Jordan. Journal of Pharmaceutical Health Services Research, 2020. 11 (3): p. 237-243.

34. خامسلو, I., et al., Contributing factors to nursing error in emergency department: A qualitative study. دانشكده مجله (حيات نهر ن بزشكى علوم دانشخاه مامايى، و برستارى), 2017.23(1): p. 17-32.

35. BRADY, A.M., A.M. MALONE, and S. Fleming, A literature review of the individual and systems factors that contribute to medication errors in nursing practice. Journal of nursing management, 2009. 17(6): p. 679-697.

36. Aronson, J.K., Medication errors: what they are, how they happen, and how to avoid them. QJM: An International Journal of Medicine, 2009. 102(8): p. 513521.

37. Hashemi, F., A. Nikbakht Nasrabadi, and F. Asghari, The obstacles of reporting nursing errors in Iran: a qualitative study. Iranian Journal of Medical Ethics and History of Medicine, 2011 .4(2): p. 53-64.

38. Ahangarzadeh Rezaei, S., et al., A survy on the nursing-related factors influencing medication error incidence. J Urmia Nurs Midwifery Fac, 2014. 12(12): p. 1088-93.

39. Ghorbanzadeh, M., et al., The Prevalence, Barriers to Medication Error Reports, and Perceptions of Nurses toward the Causes of Medication Errors in the Hospitals Affiliated to North Khorasan University of Medical Sciences, Iran. Iran Journal of Nursing, 2019. 32(117): p. 58-68.

40. Bvdlayy, H. and A. Koushki Jahromi, Sattari nasab R. learning and organizational trust as a mediator between psychological empowerment and organizational commitment rings. Management Development. 1390(75): p. 67-94. 